\title{
Loyalty Points on the Blockchain
}

\author{
Dhwani Agrawal $^{1}$, Natalia Jureczek ${ }^{1}$, Gajane Gopalakrishnan ${ }^{1}$, Margaret Natalie Guzman $^{1}$, Michael McDonald $^{1}$ \& \\ Henry Kim ${ }^{1}$ \\ ${ }^{1}$ Schulich School of Business, York University, Toronto, Ontario, Canada \\ Correspondence: Henry Kim, Schulich School of Business, York University, Toronto, Ontario M3J 1P3, Canada
}

Received: August 5, 2018

Accepted: August 28, $2018 \quad$ Online Published: August 30, 2018

doi:10.11114/bms.v4i3.3523

URL: https://doi.org/10.11114/bms.v4i3.3523

\begin{abstract}
Although some organizations are contemplating the potential impact of blockchain technology in today's economy, blockchain, itself, is quickly emerging to be a disruptive force. This is especially true in the circumstance of loyalty programs.

Blockchain is a public, digital, and distributed database solution providing decentralized management of transactional data. This technology is transforming society in ways that were previously unimaginable. Whether it be the way individuals use their phones, cars, or the healthcare system, blockchain is applicable for a variety of economic sectors and transactions. Although many may argue that blockchain is in its early development stage, it still possesses the power to revolutionize industries and consumer habits at a global scale. Through implementation of blockchain for loyalty networks, companies eliminate the limitations and inefficiencies while elevating the customer experience with secure and immediate redemption options from a variety of vendors. Despite evolving rapidly, its implementations provide better security, privacy, performance, usability, data integrity, and scalability, to name a few. Hence, blockchain is likely to entice any individual for instantaneous incentives for every purchase.

This paper aims to analyze the current, traditional loyalty programs and the challenges associated with them. It highlights how blockchain can resolve these challenges and provide a better experience. This report further explores the various types of loyalty programs that currently exist in the blockchain ecosystem and provides potential future implementations. Finally, the paper analyzes the implementation of coupons in comparison with loyalty points programs, highlighting the vast spread of blockchain implementation.
\end{abstract}

Keywords: loyalty program, blockchain, technology, decentralized database

\section{Introduction}

With the new era of rapidly evolving technological advances, blockchain proves to be another ground-breaking invention. Blockchain is a public, digitally accessible, and database solution providing decentralized management of transactional data. Various sectors of the economy, including but are not limited to healthcare, technology, accounting, consulting, and manufacturing, could use blockchain for a variety of economic transactions and record-keeping purposes. Despite the relatively new advancement of blockchain technologies, its revolutionizing power to change the industrial and consumer habits globally is remarkable. Blockchain also provides better security, privacy, usability and data integrity for its users.

Blockchain proves to eliminate the limitations and inefficiencies that companies face nowadays without the use of such technology when providing loyalty program services. By implementing blockchain, companies could elevate the customer experience with secure and instantaneous redemption from various vendors. Hence, companies could entice its customers via this technology for instantaneous rewards for every purchase.

\section{Method}

The study focused on surveying the landscape of literature and writing about blockchain applications for loyalty programs, as well as the literature on loyalty programs that currently exist in the market. Upon successful completion of the survey, a conceptual analysis of the findings was conducted which examines how blockchain can be used for establishing a loyalty points ecosystem that addresses the challenges arising from the current loyalty programs in the market. The research and analysis focused on the major categories of loyalty programs: punch cards, points, tiered, fee-based, cash back, and coalition loyalty programs. 


\section{Discussion}

\subsection{Loyalty Programs on the Traditional Platform}

The traditional loyalty programs available in today's market are very diverse. Nevertheless, despite this wide variety, the loyalty programs can be organized into a few major categories: punch cards, points, tiered, fee-based, cash back, and coalition loyalty programs.

\subsubsection{Punch Cards}

Every time a customer buys a specific good or service, the card is "punched" (Raymond, 2017). Once the card is filled out, the customer redeems the card for a free product or service (Raymond, 2017). Although technology has resulted in companies offering digital punch cards, the concept remains the same. For example, every time a customer purchases a pretzel at Wetzel's Pretzels, their phone is scanned, and the transaction is recorded on the app as a dot on the Rewards meter (Raymond, 2017). Once the customer purchases six pretzels, they are entitled to a free pretzel (Raymond, 2017).

\subsubsection{Points Loyalty Programs}

Points loyalty programs reward customers for doing a certain transaction or action. As a customer, you can “... earn points for transactions, engagement, or visits and can redeem points for rewards" (Burnett, 2017). Recently, many companies have expanded their points loyalty programs "... to reward non-transactional behaviour including downloading a mobile app, subscribing to an e-newsletter, liking a page on Facebook ..." (Burnett, 2017) among other actions that keep the customer engaged and aware of the company's offerings. This type of program is most suitable for companies with high-frequency purchases and interactions (Burnett, 2017). For example, Dick's Sporting Good has a ScoreCard Rewards program in which, for every dollar spent, customers earn one point and are eligible for a $\$ 10$ Reward once they have earned 300 points (Burnett, 2017).

\subsubsection{Tiered Points Programs}

Similarly, in tiered points programs, customers earn points based on actions or transactions. However, this program differs as customers are placed in different levels within the rewards program based on their spending and purchasing behaviour (Burnett, 2017). For example, in the Starbucks Rewards program, there are two levels members can belong to: Gold and Green ("It's Nice to Be Rewarded," n.d.). To attain Gold Level, a Green member must collect 300 stars within 12 months ("It's Nice to Be Rewarded," n.d.). Once a Gold member, customers are entitled to all the benefits they received as a Green member in addition to new exclusive benefits such as a free drink or food item once they earn 125 stars ("It's Nice to Be Rewarded," n.d.). Essentially, the bonus of this program is that it "... provides both immediate gratification with short-term attainable rewards (Burnett, 2017).

\subsubsection{Fee-Based Loyalty Programs}

Fee-based loyalty programs work in similar fashion to that of an all-you-can-eat-buffet: the customer pays a fee to enter the program but receives an "unlimited" benefit in exchange. This program encourages repeat customers as they already paid for the benefits (Customer Insight Group). At the minimum, customers can break-even, but there is the potential to obtain more benefits than paid for (Burnett, 2017). For example, Amazon Prime charges customers an annual membership and, in return, provides free express shipping (Burnett, 2017).

\subsubsection{Cash Back Loyalty Programs}

Cash back loyalty programs offer cash back on certain products and services offered by the firm. This entices customers to consistently shop at the firm to increase their savings - especially since the cash received in return makes the company's products slightly cheaper than competitors (Electronic Merchant Systems). For example, TD has a TD Cash Back Visa Infinite Card in which customers earn 3\% in cash back dollars on specific purchases and 1\% on all others ("TD Cash Back Visa Infinite Card," n.d.).

\subsubsection{Non-Monetary Rewards Program}

Rather than focusing on monetary rewards, these programs focus on customer values (Azpiral, 2017). This kind of program still engages customers, but also helps positively impact the perception of the brand by performing actions that serve the greater good (Azpiral, 2017). For example, Toms' One for One program donates a pair of shoes to a child in need for every pair purchased (Azpiral, 2017).

\subsubsection{Coalition Loyalty Programs}

Coalition loyalty programs can have properties of the programs but are often supported by multiple companies. This increases the rewards to the consumer and is therefore more attractive. For example, Air Miles points can be collected from multiple products across multiple partners (Customer Insight Group). 
The above are just the main categories of loyalty programs. Many programs have characteristics inherent to more than one category and often loyalty programs use more than one system to reward and retain customers. The common characteristics across all loyalty programs is the bestowment of beneficial value, collection and usage of consumer data, raising of switching costs for the consumer, and increasing customer retention rates. (Sharp, Byron and Anne Sharp, n.d.).

\subsection{Challenges of Traditional Loyalty Programs}

Loyalty points have various advantages that help firms to reach out to their targeted consumer base. They also help firms to ensure that they have a stable customer group so that they can generate enough revenue to continue their operations. However, there are also challenges related to running a loyalty program on the firm's side, and for the consumers who are enrolling into these programs.

\subsubsection{Company's Perspective}

From the company's perspective, it is difficult for managers to control and estimate the liabilities related to redeeming the loyalty points earned by consumers (Tierney, 2017). For example, customer claims on loyalty programs create costly liabilities on the firm's financial statements. Similarly, companies experience difficulty measuring the ROI and its effect on the net income value (Chainlink, 2018).

Another challenge for companies is finding ways to personalize the loyalty programs that will meet the needs of individual customers as broad approaches tend to fail in the long run (Burkard, 2017). Furthermore, providing loyalty points does not guarantee a secured market share. Customers with loyalty points don't directly convert into loyal customers: there will always be consumers who will tend to choose their preferred brand that might not even offer a loyalty program. Thus, loyalty points is just a way to entice loyalty in a customer (Crmsearch, 2018).

Moreover, there is a lack of integration across the channels being used for loyalty programs. Firms use online databases to issue points and have consumers go through the process to create an account, but they don't provide real-time information on customers' point collection when they sign in. Referring back to the Dick's Sporting Goods ScoreCard Program, rewards may not be available as soon as you reach 300 points because they process rewards every 30 days ("ScoreCard Questions", n.d.).

Finally, there is lack of uniformity across all channels in terms of rewards and redemption options (Crmsearch, 2018).

\subsubsection{Customer's Perspective}

As a consumer, the disadvantages of enrolling into a loyalty points program include the need to create the account and the registration process (Burkard, 2017). Some programs require the need to fill out forms and make initial contributions which can be inconvenient for consumers.

Secondly, there are many restrictions and rules placed on when and how many loyalty points can be redeemed (Burkard, 2017). Most of these limitations are not conveyed to the consumer upon initial registration or are written in fine print in the information package.

Moreover, firms don't provide a variety of reward options to which consumers can use their loyalty points. The niche offerings do not satisfy a wider group of consumers and the loyalty points often are waste (Burkard, 2017).

Another challenge with loyalty points for consumers is staying updated in terms of the changes made in rules and policies by the company (Burkard, 2017). This potentially affects the consumer's attitude and willingness to participate in the program and leads to misunderstandings and disappointment when consumers discover that they cannot use their loyalty points towards their initial preferences.

Consumers also tend to sign up for more programs than they are actually interested in which, unfortunately, results in them neglecting the points they've collected across the board. This is also attributable to the fact that some firms fail to keep consumers constantly engaged which causes consumers to eventually feel disconnected from that particular program (Burkard, 2017). This relates to the issue that customers are sometimes not provided with a way to track their loyalty points and are clueless to how long it will take to redeem their points for something of value.

Furthermore, loyalty points can potentially create switching costs for consumers who would like to switch to a competitor with a better value proposition. As a result, loyalty points cause consumers to choose a product that will reward them points although they are aware that they can receive a better offer elsewhere. For example, there are some individuals who prefer to rent vehicles from Discount Car and Truck Rentals because they will receive Petro Points at the end of their rental agreement. Since they purchase gas regularly from that specific gas station, they find this deal to be worth their time. However, there may be cheaper deals and superior service at Budget, but the loyalty points offered by Discount incentivizes them to go there instead. 
Finally, consumers tend to wait longer than expected to earn enough points for their desired product. This results in customers leaving the program earlier than expected (Crmsearch, 2018). For example, with a credit card that offers airline promotions, purchases worth thousands of dollars must be made beforehand so as to accumulate enough points to obtain a free plane ticket - this can take longer for some than others. On average, studies show that it takes about six to nine months to accrue sufficient points (Crmsearch, 2018).

\subsection{Blockchain Solutions}

Having discussed the variety of challenges regarding loyalty programs from both the firm's and customer's perspective, delving into how blockchain can be, and is, used for establishing an ecosystem to address at least some of these challenges is the next step. Singapore Airlines recently announced their adoption of a blockchain-based loyalty points program in partnership with other Singapore retailers (Shayon 2018). They will be used as a leading example in this section.

First, it is important to emphasize that blockchain is not a replacement system for a company's existing loyalty program, but instead, it is a system facilitator (Deloitte, n.d.). This means that blockchain can significantly reduce costs incurred from having loyalty programs for firms. Cost savings will appear from system management, transaction, and customer acquisition. A large portion of the high management costs stems from fraud and error. By using blockchain's platform, known for its transparency with transactions, companies like Singapore Airlines will experience much less of a burden from their loyalty program.

Furthermore, due to individuals being able to exchange points and collect all points in one wallet, customers will be able to redeem their points faster and more efficiently. This will in turn increase the number of redemption transactions, decreasing cost per transaction (Deloitte, n.d.). For Singapore Airlines, it cost them "an extra seat every time a customer redeems miles for a free flight" (Shayon, 2018). With blockchain, this will no longer be the case, especially when points handed out by Singapore Airlines can be redeemed at partnering retailers within the loyalty network. As a result of better redemption rates, the loyalty programs become more appealing to customers.

Also, companies will spend less on customer acquisition costs since blockchain-based loyalty programs will operate on social media platforms (Deloitte, n.d.). Firms adopting a blockchain-based loyalty program will also experience a smaller burden on their balance sheets. Companies in the travel industries especially (ex. airlines and hotels) must hold all their customers' unredeemed points in the form of deferred revenue (Kowalewski, McLaughlin, \& Hill, 2017). Singapore airlines, for example, holds $\$ 700$ million in deferred revenue (Shayon, 2018). With blockchain, companies can offer more redemption options to their loyalty customers, meaning they in turn will hold less liabilities on their balance sheet.

Blockchain-based loyalty programs provide many solutions directly to the consumer as well. As previously discussed, customers often wait a lot longer than expected to accumulate their points. To combat this, blockchain has the capability of providing a loyalty network. Singapore Airlines' partnership with other retailers makes it easier for customers to spend their air mile points on products outside of the airline's offerings. Furthermore, blockchain's technology facilitates the interaction between businesses in terms of the convertibility and exchange of points. By providing all consumer participants with a "digital wallet" for all their loyalty points with varying companies, they essentially hold loyalty tokens that end up being exchanged/redeemed.

\subsection{Different Types of Loyalty Points within a Blockchain Ecosystem}

As discussed, there are a few major types of loyalty programs that exist within the platform of traditional loyalty programs. Not surprisingly, with the development of blockchain technology, many of these types of loyalty programs have now been implemented within a blockchain ecosystem. Although "complete digitalization provides a great opportunity for integration of many different loyalty programs on the same platform ..." (Marshall, 2017), the loyalty programs that exist can be subcategorized and the programs provide different benefits depending on whether they pertain to a business to consumer relationship, in which customers earn loyalty coins, or business to business, which focuses more on creating a network and increasing brand recognition. 


\subsubsection{Business to Consumer}

\begin{tabular}{|c|c|}
\hline \multicolumn{2}{|c|}{ Business to Consumer } \\
\hline Customers earn loyalty & for completed transactions \\
\hline Generic Loyalty Points & Company Specific Loyalty Points \\
\hline Universal loyalty cryptocurrencies & Company specific loyalty tokens \\
\hline $\begin{array}{l}\text { Points can be redeemed with all other companies } \\
\text { with a loyalty program on that given platform or } \\
\text { can be exchanged for another cryptocurrency or } \\
\text { even fiat currency }\end{array}$ & $\begin{array}{l}\text { Points can be redeemed with the company that } \\
\text { issued the token as well as related companies and } \\
\text { partner companies }\end{array}$ \\
\hline $\begin{array}{l}\text { Examples: } \\
\text { Orioncoin } \\
\text { Elements } \\
\text { Loyyal } \\
\text { Appsolutely }\end{array}$ & $\begin{array}{c}\text { Examples: } \\
\text { Manifold Technology } \\
\text { Mobivity } \\
\text { Singapore Airlines }\end{array}$ \\
\hline
\end{tabular}

Figure 1. Business to Consumer Loyalty Points within a Blockchain Ecosystem

For consumers, loyalty points within a blockchain ecosystem accumulate in one "wallet" (Kowalewski, McLaughlin, \& Hill, 2017). Essentially consumers will earn points when they make a purchase at a company that has their rewards program on the blockchain. Depending on whether the loyalty points are company specific or generic will then determine how consumers can use those points. Figure 2 shows an example of a customer earning and redeeming loyalty points within a blockchain ecosystem.

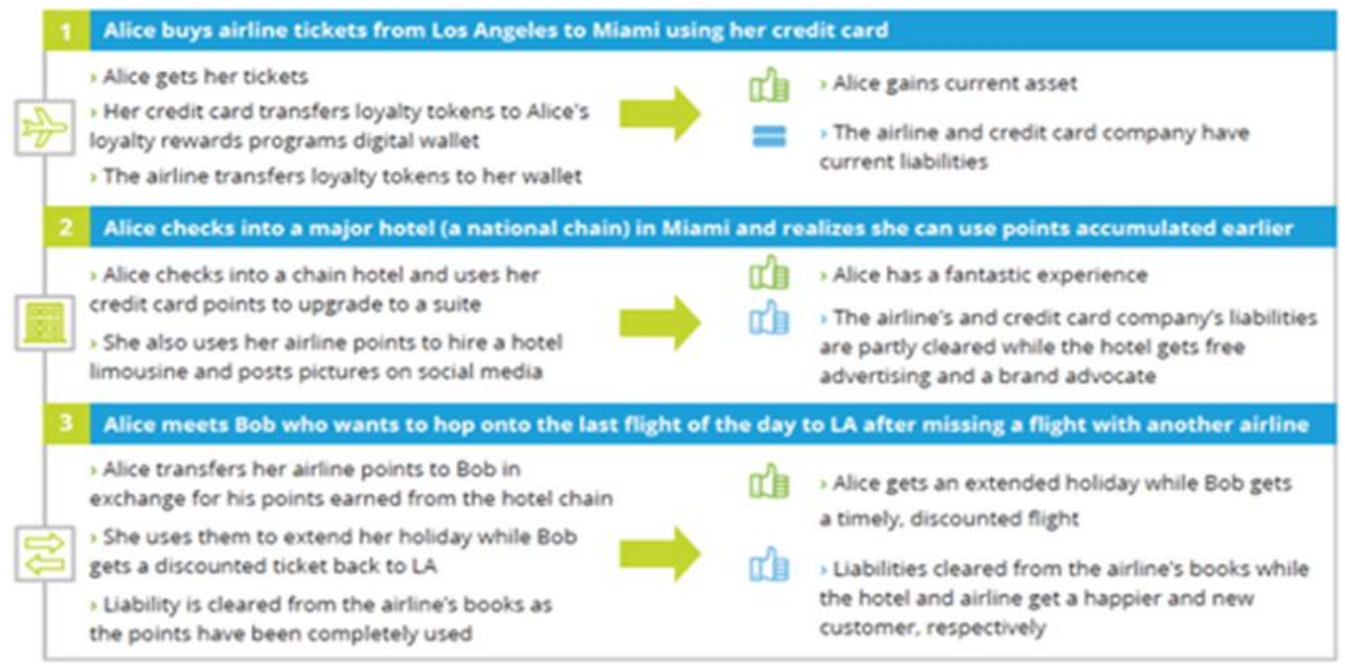

Figure 2. How a Customer Earns and Redeems Loyalty Points within a Blockchain Ecosystem

Source: Deloitte

Generic and company specific loyalty points have already been implemented in a blockchain ecosystem by both start-ups and major companies. Reward programs with generic loyalty points are ones in which users obtain universal loyalty cryptocurrencies that are accepted by all other merchants that use the same platform and can even be converted into fiat currency or other cryptocurrencies. Reward programs with company specific points allow customers to redeem their points only at the company where they earned their points or that company's partners.

Generic loyalty points offer many benefits for merchants and customers. For customers, loyalty points are very valuable as they give customers the freedom to redeem the points wherever they would like or to exchange for fiat currency or other cryptocurrencies (Adeyanju, 2017). For merchants, the ability for their rewards to provide that kind of freedom to their customers increases the value they provide to customers (Adeyanju, 2017). Reward programs with universal loyalty cryptocurrencies helps merchants reduce their balance sheet's loyalty liability (Adeyanju, 2017). Loyalty points represent a promise to the customer and are therefore recorded as a liability on the balance sheet of a company. Thanks to reward programs shifting to blockchain, "merchants could mine [the cryptocurrencies] for themselves and offer the loyalty coins to customers" (Adeyanju, 2017) while universal loyalty cryptocurrencies, specifically, further help in reducing this 
liability as merchants “... also get to accept loyalty coins that other merchants mine" (Adeyanju, 2017). Furthermore, once a customer redeems their points, the "Blockchain-based currency could be traded for fiat currency, helping them recoup, at least, some of the sales made with loyalty points" (Adeyanju, 2017).

- "Orioncoin (ORC) is the next-generation multi-tiered loyalty and marketing platform which harnesses the power of the blockchain ..." (Cointelegraph). On Orioncoin's platform, customers earn transferable cryptocurrencies, ORC, which they can redeem for goods and services or cash out (Serafino, 2018). Furthermore, "the Orioncoin platform offers credit cards for international usage, as well as the ability for users to move funds to fiat currencies and then shift them to a conventional bank account, all within a single platform" (Buck, 2017). Essentially, Orioncoin's platform is comparable to credit cards ".... that allow members to earn transferable points and use them with their airline and hotel partners, or buy gift cards, electronics and other stuff' (Serafino, 2018) although Orioncoin's platform goes beyond as it is neither limited in scope nor centralized.

- Elements is another example of an universal loyalty cryptocurrency (Coleman, 2017). Similar to Orioncoin, all the merchants on this platform accept Elements loyalty coins and the coins can be exchanged for fiat and cryptocurrencies ("Blockchain Based Loyalty Program," n.d.). As "Elements aims [to transform] the conventional loyalty/rewards programs to Blockchain based loyalty programs" ("Blockchain Based Loyalty Program," n.d.), it has given its users the freedom to store the coins in any e-wallet so that all coins can accumulate in one place and not be diluted (Coleman, 2017). Furthermore, Elements provides even more benefits to the merchants that use the platform as "... merchants can acquire 'Elements' coins for free by mining the currency, thus reducing the cost of liability" (Coleman, 2017). To generate the blocks, this platform uses proof of work (PoW) in which users are required "... to perform some type of work to participate" (Coleman, 2017). For their participation, miners can earn 50 Elements coins and/or a fraction of the block's transaction fee (Coleman, 2017).

- Loyyal, formally Ribbit.me (EconoTimes, 2016), “... is the universal loyalty and rewards platform, built with Blockchain and smart contract technology" ("Loyyal”, n.d.). This platform operates across industries and allows for multi-branded, rather than individually branded, rewards (Anonymous, 2017). This means that, for example, airline/bank or airline/retailer/consumer reward programs can exist (Anonymous, 2017). On this platform, "loyalty incentification can now flow up the relationship network from customer to merchant as easily as it moves down, sideways, or other directions" (Anonymous, 2017). Not surprisingly, PWC, Machine Colony, and Deloitte are already some of the major companies that have already partnered with Loyyal (Anonymous, 2017). Loyyal does not currently have its own token cryptocurrency “... and companies operate their own branded programs on a permissioned, private Blockchain” (MacKenna, 2018).

- Appsolutely Inc. is an universal loyalty platform that has been launched in the Philippine market “.... to centralize all loyalty programs" (Esmael, 2017). LoyalCoin, the digital token used on this platform, is earned from one retailer and "... can be easily used to pay for products from other brands" (Esmael, 2017). "The company has since evolved into an omnichannel platform provider, with a solutions portfolio including e-commerce, digital marketing, web and app development, and logistics" ("Loyalcoin," n.d.).

Company specific loyalty programs are not common on the blockchain platform as "... private Blockchains make the application more complex, less efficient and more expensive than a centralized database ...” (Willms, 2017). For the users, private blockchains will also come with challenges as they “... will have difficulty accessing it from the public internet" (Willms, 2017). Not surprisingly, there are "... a number of experts [that claim] that private permissioned Blockchains defeat most of the purpose of using Blockchain technology" (Willms, 2017). Nevertheless, there are companies that use private blockchains as this kind of platform allows for immediate interaction between businesses and their customers (Willms, 2017).

- Manifold Technology has a private blockchain that they hope will become the standard for the banking industry (Willms, 2017). Their platform, “... Manifold Liquidity Platform, provides private high-security blockchains for financial institutions and their customers together with real-time analytics ..." (Levich, 2016). Manifold has already partnered with RBC and “... is currently working with a large Asia-Pacific bank and several healthcare multinationals to develop an application that will offer greater data privacy and security” (Willms, 2017). In the case of RBC, the partnership's “... goal is to let users of RBC's credit cards collect and redeem rewards points instantly after completing qualifying transactions at retail stores and other merchants" and will essentially replace the rewards system they currently have in place (Nash, 2017). To be able to collect rewards data in real-time, “... RBC plans to add its blockchain system to the payment processing network it already runs to complete debit- and credit-card transactions for merchants" (Nash, 2017). 
- Following the partnership between Mobivity and Chanticleer Holdings, the CEO of Mobivity, Dennis Becker, has stated that "Eating a burger is now a way to mine for cryptocoins! Every meal enjoyed at any Chanticleer Holdings brand will accrue currency for the consumer that can be used for future meals or traded with other consumers" (Mobivity Holdings Corp., 2018). As Chanticleer operates BGR, Hooters, Little Big Burger, American Burger Company, and Just Fresh, the cryptocurrency, Mobivity Merit, can be used across all the brands (Mobivity Holdings Corp., 2018). Thanks to the loyalty program, customers can now earn points at BGR with every meal and redeem the points at Just Fresh. Going beyond the traditional loyalty programs, Mobivity allows customers to transfer points among themselves (Mobivity Holdings Corp., 2018).

- Singapore Airlines is going to "... launch a digital wallet for its frequent-flyer KrisFlyer program [...] that will be powered by a private blockchain" (Zhao, 2018) that the company owns (Anonymous, 2018). Once launched, Singapore Airlines will have created "... the world's first blockchain-based airline loyalty digital wallet ..." (Kang, 2018). On this platform, when " ... a customer redeems 50,000 miles for a business class seat, for example, the company could then immediately sell the miles in the market for an equivalent amount of cash. This implies that the traders of Singapore Airlines's "miles cryptocurrency" would be funding the company's loyalty program (or a discount marketing campaign), reducing the firm's financial burden to basically zero" (Kang, 2018). Users of the platform will be able to "... spend their air miles at retail partners for point-of-sale transactions" (Zhao, 2018).

\subsubsection{Business to Business}

While a blockchain eco-system allows consumers to accumulate tokens in a digital wallet, the business to business platform differs as it focuses more on creating a network and, through these connections, increasing brand recognition (figure 3 illustrates an example of how this network would look).

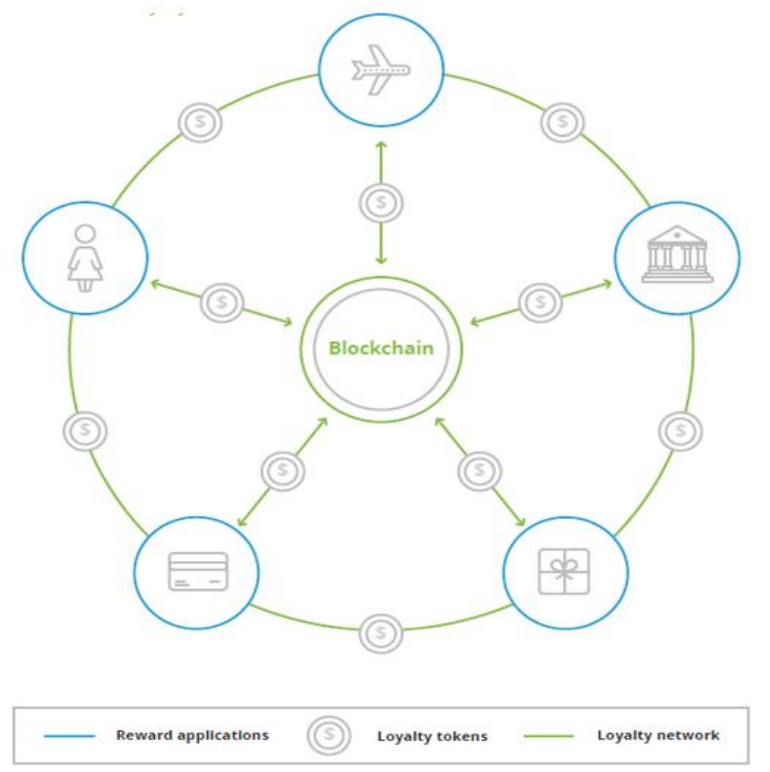

Figure 3. Blockchains-Based Loyalty Networks

Source: Deloitte

First, Loyalty points within a blockchain ecosystem provide businesses with a platform to connect easily with a variety of organizations and eliminate the role of a middleman (Smith, 2017). Specifically, this network "... facilitates their interactions, especially the converting and exchanging of points" (Smith, 2017).

Companies with well-developed loyalty programs on the blockchain ecosystem have a value-added service that they can offer to clients (Smith, 2017). For example, a bank will now be able to offer its small-business clients "... access to its loyalty rewards interlinked network [and] the opportunity to join the overall interlinked network on the [client's] own terms through a tailored rewards application" (Smith, 2017). Evidently, this increases brand recognition for the small-business as "... a local coffee shop that was afforded entry into the network by its bank could offer its customers opportunities to use their coffee points toward accommodations at a hotel in another state in which it previously had little to no brand recognition" (Smith, 2017).

Due to its simplicity, a blockchain ecosystem will make it easier to create and maintain these partnerships, between both large and local businesses, which will increase the redemption options available (Kowalewski, McLaughlin, \& Hill, 
2017). This will benefit the businesses involved in these networks as it will help them decrease the liability balance that the traditional loyalty programs created (Kowalewski, McLaughlin, \& Hill, 2017).

Essentially, the blockchain ecosystem will facilitate business to business interactions and will make it easier to expand redemption options. "On Loyyal's network, [for example,] two companies that want to accept each other's points can agree on the terms of exchange, create smart contracts that build the exchange terms into a programming language, and enable exchanges to occur under customer control with no centralized management or reconciliation" (MacKenna, 2018).

3.4.3 Other Types of Loyalty Points within a Blockchain Ecosystem
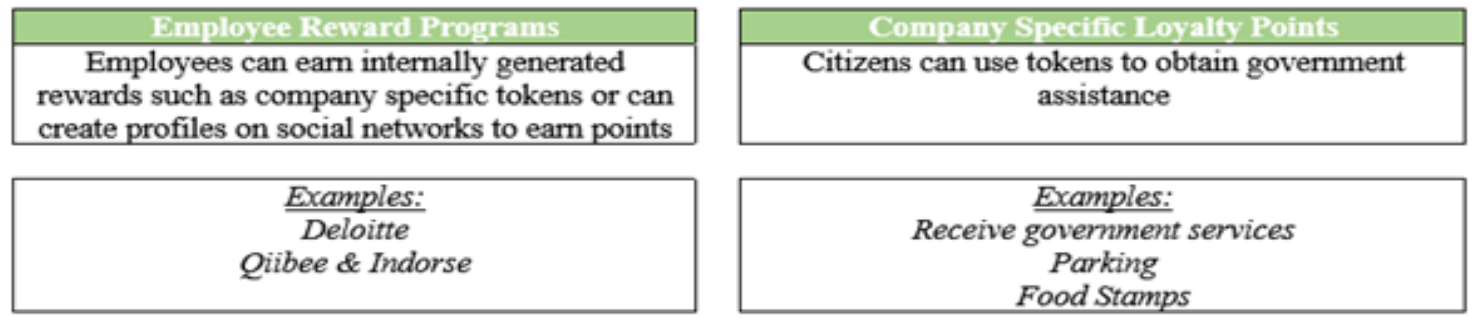

Figure 4. Other Types of Loyalty Points within a Blockchain Ecosystem

Although business to consumer loyalty programs are the most common within a blockchain ecosystem, there are many different types that exist!

- Employee Reward Programs. With the emergence of blockchain technology, companies have even begun to shift their employee reward programs onto the blockchain ecosystem. Deloitte currently has implemented a rewards system in which employees receive DCoins while Qiibee and Indorse have partnered to create a platform that employers will be able to customize for their employees.

- Deloitte. Besides partnering with Loyyal, “... Deloitte is piloting blockchain on an internal rewards program known as DCoins ...” (MacKenna, 2018). Per Eric Piscini, a Deloitte principal, “... blockchain-based rewards are suitable for employee programs, as well as customer programs in retail, credit cards, travel and hospitality" (MacKenna, 2018).

- Qiibee \& Indorse. Qiibee, a decentralized blockchain-based loyalty ecosystem, and Indorse, a social network for professionals, entered into a partnership to use blockchain technology as a means to reward employees (Warner, 2017). Companies who use the Qiibee platform will receive “... a developer kit with everything needed to create their own customized loyalty platform (Warner, 2017). Furthermore, by partnering with Indorse, Qiibee has taken a different approach to the way points are earned: “... users will build their profiles and profit from their reputation by sharing their data and evaluating the claims of other users" (Warner, 2017). This platform will use a mechanism similar to the Proof of Stake in which "... the Indorse Score allows moderators to stake their reputation on a claim or indorsement ..." (Warner, 2017).

- Citizen Reward Programs. Using blockchain technology has also gone beyond business to consumers or even business to business, as loyalty programs on the blockchain can be used for citizens that receive government assistance. Reward programs within a blockchain ecosystem would “... improve citizens' ability to use and receive government services, from parking to food stamps" (MacKenna, 2018) similar to the way that consumers receive tokens from businesses.

\subsubsection{Blockchain Implementation Proposals for Traditional Loyalty Programs That Have Not Been Implemented Yet}

Based on the discussion above, cash back, point, and coalition loyalty programs have been implemented on the blockchain ecosystem. For those that don't yet exist, this report outlines proposals on how they could be implemented.

- Tiered Points Program. The loyalty programs previously discussed only had one level of members. To implement a tiered program, companies can offer their frequent purchasers more tokens per dollar spent. In the Starbucks example discussed above, customers were given a free item every 125 stars. In the blockchain ecosystem, points are not used to keep you in a reward program, but are essentially cryptocurrencies. Therefore, this system is already in place as customers can redeem their points for items at the company from which they received the loyalty point and others, depending on whether that company provides generic or company-specific points. Having a tiered points program can be a source of competitive advantage for companies in the blockchain 
ecosystem as customers tend to pick loyalty programs where their commitment will result in obtaining greater benefits than less loyal customers.

- Fee-Based Loyalty Program. Currently the loyalty programs implemented within the blockchain ecosystem are free for customers to join and only require customers to take part in transactions to earn points. A fee-based loyalty program can be another source of competitive advantage as customers are more willing to choose a company that provides them with an unlimited value of tokens at their disposal to redeem for discounts or free services (ex. shipping). Although this would be harder to implement, it may be practical if a new kind of token is invented: a token that can be used an unlimited amount of times and for an unlimited amount of monetary value with that specific company (for a specified period of time, for specified services and one which cannot be transferred).

- Non-Monetary Rewards Programs. Programs currently implemented in the blockchain ecosystem provide customers with a monetary reward as these tokens are essentially cryptocurrencies and can be redeemed or transferred to other cryptocurrencies or fiat currencies. However, that does not mean that non-monetary rewards cannot be given. In the blockchain ecosystem, this would work best on a private blockchain where the company gives their own unique tokens for each purchase made. These tokens would be unique as their properties would indicate that they cannot be redeemed as a currency by the customers, but must be transferred to an account owned by a nonprofit organization (NPO). The NPO's account would then be able to exchange the tokens for a free pair of shoes, as in the case of Toms. Implementing this kind of program would increase transparency and customers would be more engaged by actually transferring the token that provides a child in need with a free pair of shoes, for example.

- Business to Business Loyalty Tokens. Blockchain-based loyalty programs are beneficial as they help connect businesses that would not have otherwise been connected. To incorporate a platform where businesses can get loyalty points (essentially cryptocurrencies) from another business in their capacity as a business as opposed to a consumer, partnerships would have to be put in place. That is, there could be partnerships that state that if a customer uses tokens it obtained from Company A to redeem at Company B as a result of some sort of referral program (ex. Company A promotes Company B's goods), Company B could award Company A loyalty points for attracting new clients to them.

\section{Results}

\subsection{Blockchain Implementation Guide for Companies}

As blockchain technology becomes more integrated in how we do business and as more loyalty programs, as discussed above, are created on the blockchain ecosystem, more companies will be transferring their loyalty programs. Figure 5 shows the steps a company must take when implementing their loyalty points on the blockchain with the first step being a buy or make decision.

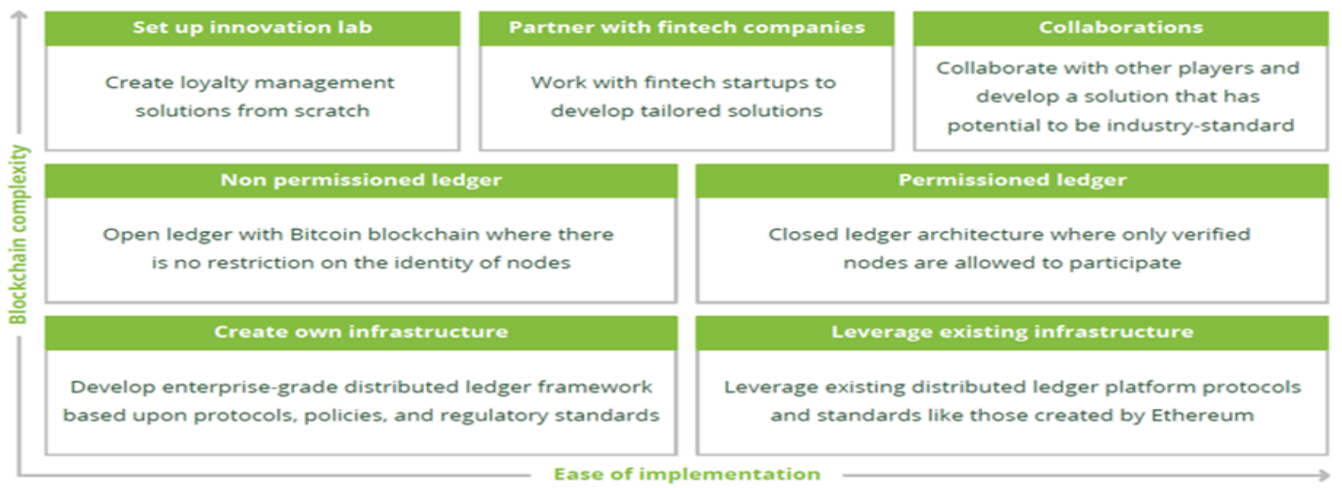

Figure 5. Implementation Roadmap

Source: Deloitte

\subsection{Usage of Coupons versus Loyalty Points}

\subsubsection{The Purpose of Coupons and Loyalty Programs}

There are two types of loyalty and rewards programs. Firstly, there are the aforementioned loyalty programs that reward a customer's loyalty. Second, there are coupons which incentivize consumers into a single transaction by providing a one-time discount or cash-back, leaving no residual loyalty relationship afterwards (Adeyanju, 2017). 
The first major difference between the two is that loyalty programs are not limited to a one-time use and can be used with other reward programs. Although coupons provide similar incentives, they are a single use, cash-back rewards program that cannot be combined with other coupons. Contrary to loyalty programs, coupons usually have an expiry date after which they cannot be used. In either case, the benefits offered encourage customers to form a relationship with a business, even if only for one transaction, with the hope of winning a repeat customer. Nevertheless, it is evident that loyalty programs are better at retaining customers as they reward customers for repeat purchases. For example, in the tiered loyalty program, customers with frequent purchases obtain more benefits than others; therefore, repeat customers are highly encouraged.

Next, due to the requirement for customers to register for loyalty programs, businesses are able to record and gain valuable data. On the contrary, most coupons do not require any information from customers in order to be used making coupons less valuable in comparison.

Finally, as a direct brand-to-consumer program, a coupon has the added short-term goal of liquidating excess inventory faster while loyalty programs are more oriented about transactions going into the long-term.

\subsubsection{The Impact of Blockchain on Industries}

Loyalty programs have proliferated across travel, retail, financial services, and other economic sectors (Kowalewski, McLaughlin, \& Hill, 2017). However, the industries that have shifted their loyalty programs to the blockchain ecosystem are those more generic in nature while the more intricate industries still must conduct considerable development. One such industry would be agriculture, as opposed to the retail industry where the development of loyalty programs is extensive.

Coupons. Currently, there are not many industries that implement the use of coupons within the blockchain ecosystem. Contrary to loyalty points, coupons are used very selectively, mainly in the retail industry.

Recommendation. Companies should implement coupons and loyalty programs on the blockchain ecosystem within manufacturing, retailing, wholesaling, banking, and travel sectors as the customers of these industries would benefit most from a decentralized platform. On the other hand, a loyalty program on the blockchain ecosystem can be implemented in the health industry. For example, donors would receive non-monetary tokens each time they donated blood. Blockchain would then help keep track of the donor's information and the number of times they donated or redeemed their tokens for blood.

\subsection{Impact of Improving Technology}

The main issue that businesses face when it comes to discounts, rewards, and coupons is to make them both relevant to the customer and profitable for the company. Often customers see promotions as being more of a hassle than they are worth since they often require further spending to be redeemed.

From the business perspective, these promotions are hard to get right. Not valuable enough means they are ignored by the customer. Too valuable, the business may incur a significant loss that does not lead to increased sales. Generous promotions often attract customers that are unlikely to convert into loyal and repeat customers (Kowalewski et al., 2017).

The existing situation makes it very difficult for businesses to hit the sweet spot where discounts lead to increased profits in the medium- to long-term. In the old days, it was the labor of collecting coupons. Now customers must decide if they will suffer the hassle of downloading and using new apps specific to each business. This lack of involvement makes it harder for companies to gather data for market research and customer targeting. A coordinated approach could drive a more comprehensive and coordinated collection of data.

The technology implemented for these programs are new and are continuously improving to ensure new features address these issues. With blockchain as the new, emerging technology, implementation makes these programs more convenient, but the implementation may not be all that simple.

Loyalty Points Programs. The biggest drawbacks to implementing blockchain for loyalty programs include high initial development and implementation costs and possible loss of control over data (Adeyanju, 2017).

First, blockchain-based loyalty rewards programs can be expensive to build due to substantial start-up costs of such a relatively new technology (Kowalewski et al., 2017). However, there are new platforms and solutions specifically designed for implementing loyalty programs becoming available which will help make the switch easier.

Furthermore, with the implementation, companies could lose control over their data which they currently have with their existing, conventional loyalty platforms.

Fortunately, there are various ways companies are combating these challenges. For example, Elements has already created a secure platform for loyalty points on the blockchain ecosystem. Elements uses X11, a chained hashing algorithm that is 
used for PoW calculations which creates proof of the transaction and is trying to become a universally accepted loyalty cryptocurrency (Adeyanju, 2017). Since this is a "PoW" cryptocurrency, it can be mined by anyone who has a computer with the processing power required to mine cryptocurrencies. Miners will be rewarded with some of these cryptocurrencies for the completed work. Miners can then use the loyalty coins for everyday shopping (Adeyanju, 2017). Blockchain also addresses the former issues as it is a decentralized network: it can decentralize and unify the fragmented loyalty rewards space. Thus, it makes the reward points more valuable to customers, enticing repeat purchases, and possibly offsetting the company's initial implementation costs.

Coupons. Some of the many problems of fraud that affect traditional e-coupon systems are by the means of double redemption and repudiation. Coupon fraud is a very big issue as unsecured coupons could be copied ad-infinitum or tampered to redeem a product at the wrong price or even another product. Brands can lose control of the promotion they run with potential brand-image or financial risks (O’Connor, 2018).

In the new retail era, countless businesses overwhelm their customers with information from e-commerce APPs, promotional texts, and e-mail. Unfortunately, the large costs required make consumers unwilling to pay attention to small promotions that they are bombarded with.

Moreover, with the proliferation of false promotions and induced fraud, the lack of normative marketing activities and low transparency, post-consumer points not being redeemed, the phenomenon of isolated islands of value, user data being abused, and unauthorized sales, a customer to customer (C2C) e-coupon platform is lacking (Kowalewski et al., 2017).

Recommendation: Implement Smart Contract Technology for Coupons. At the present, many coupons are limited by merchants who set various conditions for use which makes it a particularly serious situation on a small platform. The user is holding coupons with expectations of great benefits, but cannot enjoy the discount, which is business-to-user deceptive marketing. Fortunately, trading platforms enables free flow of coupons and points, to ensure maximization of their value. The goal is to create a decentralized e-commerce coupon ecosystem and a platform that offers remittance services for points as this will maximize the value of unused coupons and enables users who need coupons to experience a good shopping experience (O’Connor, 2018).

\section{Conclusion}

Blockchain-based technology plays a crucial role in tracking coupons and other promotions as everything is transparent and the information cannot be duplicated due to the smart contract model. With the help of blockchain, businesses can authenticate the coupons and promotion codes to ensure that customers are properly using them. Therefore, blockchain addresses the difficulty of maintaining a track record of all the coupons by using decentralized databases for billions of coupons, their issuance, their respective user's ownership, and their redemption, all while preventing fraud.

Through the above measures, companies can track the historical price of goods, eliminate fraud, and conduct real-time coupon tracking to tap the potential value. The coupon system based on smart contracts will ensure the value of the coupons while the decentralized trading platform will allow coupon points to flow freely (O'Connor, 2018).

\section{References}

Blockchain Based Loyalty Program. (n.d.). Retrieved from https://theelements.io/

Blockchain, Coupons and Rebates: How The Blockchain is Going To Change The Way You Shop and Mine.... (2018). Medium.

Retrieved

from

https://medium.com/@CBT_TOKEN/blockchain-coupons-and-rebates-how-the-blockchain-is-going-to-change-the -way-you-shop-and-mine-e9c84f1eebde

CausePlay Review - VCB Virtual Cash Back Coupon \& Rewards Blockchain?. (2018). Bitcoinexchangeguide. Retrieved from https://bitcoinexchangeguide.com/causeplay/

Chainlink. (2018). 5 Common Problems With Loyalty Marketing Programs. Retrieved from https://chainlinkmarketing.com/5-common-problems-with-loyalty-marketing-programs/.

Crmsearch. (2018). How to Create Loyalty Programs That Work. Retrieved from http://www.crmsearch.com/loyalty-risks.php.

Deloitte (n.d.). Making Blockchain Real for Customer Loyalty Rewards Programs.

Glossary, L., \& Loyalty Program. (2011). Electronic Merchant Systems. Retrieved August 18. Loyalty Card: A plastic card that identifies participants in a loyalty program. Loyalty programs reward cardholders with benefits as they buy more merchandise.

Insights. (2018). Blockc60events.com. Retrieved from http://blockc60events.com/main/insights_profile6

It's Nice to Be Rewarded. (n.d.). Retrieved from https://members.starbucks.ca/ 
Loyalcoin. (n.d.). Retrieved from https://loyalcoin.io/

Loyyal. (n.d.). Retrieved from http://www.loyyal.com/

Loyyal - Smart Contract Blockchain Internet Loyalty Rewards? (2017, August 30). Retrieved from https://bitcoinexchangeguide.com/loyyal/

The Rouge Project is Carrying The Billion Dollar Coupon Space Into The Future. (2018). Global Coin Report. Retrieved from https://globalcoinreport.com/rouge-project-carrying-billion-dollar-coupon-space-future/

ScoreCard Questions. (n.d.). Retrieved from https://myscorecardaccount.com/FAQScoreCard.aspx

Sharp, B., \& Anne, S. (1997). Loyalty Programs and Their on Repeat-Purchase Loyalty Patterns", International Journal of Research in Marketing, 14(5), 473-86. https://doi.org/10.1016/S0167-8116(97)00022-0

TD Cash Back Visa Infinite Card. (n.d.). Retrieved from https://www.td.com/ca/en/personal-banking/products/credit-cards/cash-back/cash-back-visa-infinite-card/

MEDICI Team (2015, October 27). Private Blockchain Platform to Enable Revolutionary Rewards Program Experiences. MEDICI.

https://gomedici.com/private-blockchain-platform-to-enable-revolutionary-rewards-program-experiences/

Levich, A. (2016, January 7). Four new blockchain partners join Microsoft Azure. Coinfox. http://www.coinfox.info/news/4344-four-more-blockchain-partners-joining-microsoft-azure

EconoTimes. (2016, April 26). Blockchain Startup Ribbit.me Rebrands As Loyyal. EconoTimes. Retrieved from https://www.econotimes.com/Blockchain-Startup-Ribbitme-Rebrands-As-Loyyal-200254

Burnett, S. (2017, January 25). What are the Different Types of Loyalty Programs. Customer Insight Group. https://www.customerinsightgroup.com/loyaltyblog/brand-loyalty/what-different-types-loyalty-programs

Smith, K. (2017, March 7). Using Blockchain for Loyalty Rewards. Retrieved from http://deloitte.wsj.com/cmo/2017/03/07/using-blockchain-for-loyalty-rewards/

Kowalewski, D., McLaughlin, J., \& Hill, A. (2017, March 14). Blockchain Will Transform Customer Loyalty Programs. Harvard Business Review. https://hbr.org/2017/03/blockchain-will-transform-customer-loyalty-programs

Azpiral. (2017, March 29). Non-Monetary Loyalty Strategies- No Mon can still be fun. Azpiral. https://www.azpiral.com/non-monetary-loyalty-strategies/

Nash, K. S. (2017, March 31). RBC Building Customer Rewards System on Blockchain. The Wall Street Journal. https://blogs.wsj.com/cio/2017/03/31/rbc-building-customer-rewards-system-on-blockchain/

Willms, J. (2017, April 18). This Startup Put RBC's Rewards on the Blockchain. BitCoin Magazine. Retrieved from https://bitcoinmagazine.com/articles/startup-beat-out-ibm-put-rbcs-rewards-program-blockchain/

Burkard, K. (2017, May 30). 10 Reasons Customers are Abandoning Your Loyalty Program (and How to Save Them). https://blog.smile.io/why-customers-abandon-your-loyalty-program.

Ksireddy, P. (2017, July 5). Bitcoin, Ethereum, Blockchain, Tokens, ICOs: Why should anyone care? Hacker Noon. https://hackernoon.com/bitcoin-ethereum-blockchain-tokens-icos-why-should-anyone-care-890b868cec06

Hall, C. (2017, July 18). Blockchain for Customer Loyalty Rewards Programs. Cutter. https://www.cutter.com/article/blockchain-customer-loyalty-rewards-programs-496201

Tierney, J. (2017, July 18). Biggest Challenge Loyalty Programs Face Today? Managing Redemption Liability of Rewards.

Retrieved

from https://www.loyalty360.org/content-gallery/daily-news/biggest-challenge-loyalty-programs-face-today-man.

Marshall, A. (2017, September 29). Companies Applying Blockchain for Better Loyalty Programs. Cointelegraph. https://cointelegraph.com/news/companies-applying-blockchain-for-better-loyalty-programs

Raymond, K. (2017, October 4). The 4 Most Common Types of Loyalty Programs. WPromote. https://www.wpromote.com/blog/4-common-types-loyalty-programs/

Coleman, L. (2017, October 16). ELEMENTS: A Cryptocurrency Serving As A Universal Loyalty Rewards Program. $C C N$. https://www.ccn.com/elements-cryptocurency-serving-universal-loyalty-rewards-program/

Esmael, M. (2017, October 19). Appsolutely introduces universal loyalty program. The Manila Times. http://www.manilatimes.net/appsolutely-introduces-universal-loyalty-program/357392/

Staff, I. (2017, November 15). Loyalty Program. Investopedia. Retrieved from 
https://www.investopedia.com/terms///loyalty-program.asp

Pollack, R. (2017, November 25). Blockchain \& Loyalty Rewards Programs: Turning the Page. Prague Post. Retrieved from https://www.praguepost.com/technology/blockchain-loyalty-rewards-programs

TheCoinTelegraph. (2017, November 29). This new startup uses blockchain technology to offer discounts and promotions. The Next Web. $\quad$ Retrieved from https://thenextweb.com/money/2017/11/29/this-new-startup-uses-blockchain-technology-to-offer-discounts/

Adeyanju, C. (2017, December 8). How a Loyalty Program on Blockchain Works, Explained. Cointelegraph. https://cointelegraph.com/explained/how-a-loyalty-program-on-blockchain-works-explained

Warner, M. (2017, December 14). Blockchain Loyalty Ecosystem Partnership Enables Employee Rewards. Chain Finance. $\quad$ Retrieved from http://www.chain-finance.com/2017/12/14/blockchain-loyalty-ecosystem-partnership-enables-employee-rewards/

Buck, J. (2017, December 26). Blockchain Will Make Loyalty Programs Actually Work, Says Orioncoin CEO. Cointelegraph. https://cointelegraph.com/news/blockchain-will-make-loyalty-programs-actually-work-says-orioncoin-ceo

Charlebois, S. (2018, January 1). How Blockchain Technology Could Transform the Food Industry. RETAIL INSIDER. from https://www.retail-insider.com/retail-insider/2018/1/how-blockchain-technology-could-transform-the-food-industr y

Mobivity Holdings Corp. (2018, January 2). Chanticleer Holdings to Deploy Mobivity's Blockchain Technology to Power Cryptocurrency Rewards Program. Retrieved from https://globenewswire.com/news-release/2018/01/02/1277006/0/en/Chanticleer-Holdings-to-Deploy-Mobivity-s-B1 ockchain-Technology-to-Power-Cryptocurrency-Rewards-Program.html

Avdyusheva, Y. (2018, January 16). Orioncoin Further Unveils Loyalty Program Blockchain Platform. Cointelegraph. https://cointelegraph.com/news/orioncoin-further-unveils-loyalty-program-blockchain-platform

What the Hell Is Blockchain Marketing Anyway? (2018, January 18). Ebulkemaimarketing Blogs and updates. Retrieved from http://blog.ebulkemailmarketing.com/what-the-hell-is-blockchain-marketing-anyway/

MacKenna, J. (2018, February). Blockchain loyalty programs next wave of customer experience. SearchCRM. $\mathrm{http} / / /$ searchcrm.techtarget.com/feature/Blockchain-loyalty-programs-next-wave-of-customer-experience

Anonymous (2018, February 05). KrisFlyer To Launch World's First Blockchain-Based Airline Loyalty Digital Wallet. Retrieved from https://www.singaporeair.com/en_UK/sg/media-centre/press-release/article/?q=en_UK/2018/January-March/ne051 8-180205

Zhao, W. (2018, February 5). Singapore Airlines to Launch Blockchain-Based Loyalty Wallet. CoinDesk. Retrieved from https://www.coindesk.com/singapore-airlines-launch-blockchain-based-loyalty-wallet/

Serafino, M. (2018, February 7). Orioncoin Working on a Blockchain-Enabled Loyalty Program Platform. Wallet Weekly. Retrieved from https://www.walletweekly.com/orioncoin-loyalty-blockchain/

O'Connor, M. (2018, February 12). Coupons on The Blockchain Using Smart Contracts. Medium. https://medium.com/@matthewoconnor_20597/couponone-coupons-on-the-blockchain-using-smart-contracts-de9c 08d3506f

Kang, D. (2018, February 15). Is Singapore Airlines' blockchain-based loyalty program a good move for consumers and the company? The Drum. http://www.thedrum.com/opinion/2018/02/15/singapore-airlines-blockchain-based-loyalty-program-good-move-co nsumers-and-the

\section{Copyrights}

Copyright for this article is retained by the author(s), with first publication rights granted to the journal.

This is an open-access article distributed under the terms and conditions of the Creative Commons Attribution license which permits unrestricted use, distribution, and reproduction in any medium, provided the original work is properly cited. 